\title{
Smoking is associated with an increased risk of type 2 diabetes but a decreased risk of autoimmune diabetes in adults: an 11-year follow-up of incidence of diabetes in the Nord-Trøndelag study
}

\author{
S. Carlsson ${ }^{1}$ K. Midthjell ${ }^{2}$ V. Grill ${ }^{3,4,5}$ \\ ${ }^{1}$ Division of Epidemiology, Stockholm Centre of Public Health and Department of Epidemiology, Institute of Environmental \\ Medicine, Karolinska Institute, Stockholm, Sweden \\ ${ }^{2}$ HUNT Research Centre, Institute of Community Medicine, Norwegian University of Science and Technology, Verdal, Norway \\ ${ }^{3}$ Department of Molecular Medicine, Karolinska Institute, Stockholm, Sweden \\ ${ }^{4}$ Institute of Cancer Research and Molecular Medicine, Norwegian University of Science and Technology, Trondheim, Norway \\ ${ }^{5}$ Department of Internal Medicine, University Hospital of Trondheim, Trondheim, Norway
}

\section{Abstract}

Aims/hypothesis. We compared the association between smoking habits and later occurrence of type 2 diabetes on the one hand and between smoking and diabetes with autoimmunity on the other hand.

Methods. We used data from a prospective study of 11-year cumulative incidence of diabetes in the NordTrøndelag Health Survey.

Results. Confirming previous reports, heavy smoking ( $\geq 20$ cigarettes per day) carried an increased relative risk (RR) of type 2 diabetes $(n=738, \mathrm{RR}=1.64,95 \%$
CI: 1.12-2.39). In contrast, smoking reduced the risk of latent autoimmune diabetes in adults (LADA) and of traditional type 1 diabetes (LADA $n=81, \mathrm{RR}=0.25$, 95\% CI: $0.11-0.60$; type 1 diabetes, $n=18, \mathrm{RR}=0.17$, 95\% CI: 0.04-0.73).

Conclusions/interpretations. The results indicate that nicotine influences autoimmune processes in human diabetes.

Keywords Glutamic acid decarboxylase · Insulin secretion - Latent autoimmune diabetes in the adult . Nicotine $\cdot$ Smoking Type 1 diabetes $\cdot$ Type 2 diabetes

\section{Introduction}

Several epidemiological studies indicate that smoking is a risk factor for type 2 diabetes (reviewed in [1]). To our knowledge, however, it has not been investigated whether smoking affects the risk of latent autoimmune diabetes in adults (LADA) and of traditional type 1 diabetes in a manner similar to the way it affects type 2 diabetes.

Received: 8 April 2004 / Accepted: 28 July 2004

Published online: 19 November 2004

C) Springer-Verlag 2004

V. Grill (

Department of Internal Medicine,

University Hospital of Trondheim, 7006 Trondheim, Norway

E-mail: valdemar.grill@medisin.ntnu.no

Tel.: +47-73-868386, Fax: +47-73-867546

Abbreviations: Anti-GAD, GAD antibodies - LADA, latent autoimmune diabetes in the adult $\cdot \mathrm{RR}$, relative risk

Conflict of interest: None
The Nord-Trøndelag Health Survey is a large, population-based, prospective study where incident cases of diabetes were classified according to clinical history, C-peptide and presence or absence of GAD antibodies (anti-GAD). In this cohort, we investigated the association between smoking and the cumulative incidence (risk) of type 2 diabetes and of autoimmune diabetes respectively during an 11-year follow-up.

\section{Subjects and methods}

Nord-Trøndelag Health Survey. From 1984 to 1986, all inhabitants of the Norwegian county of Nord-Trøndelag who were aged 20 years or older were invited to take part in the Nord-Trøndelag Health Survey $(n=85,100)[2,3]$. The survey featured a clinical examination, including measurements of height, weight and blood pressure, and questionnaires with questions on current health, diabetes, and lifestyle factors such as smoking, alcohol consumption, physical activity, dietary habits and psychosocial conditions. Of those invited, $90.3 \%$ participated $(n=76,885)$.

Between 1995 and 1997 a second, similar health survey $(n=92,703)$ was conducted in Nord-Trøndelag, again including all subjects aged 20 years or older. The overall response rate in this follow-up investigation was $71.3 \%(n=65,258)$. 
Table 1. Baseline characteristics, Nord-Trøndelag Health Study 1984-1986

\begin{tabular}{|c|c|c|c|c|}
\hline & Subjects not reporting diabetes & Type 2 diabetes & LADA & Type 1 diabetes \\
\hline Number & 37,968 & 738 & 81 & 18 \\
\hline Age, mean (SD) & $46.3(14.3)$ & $57.5(11.1)$ & $57.0(11.6)$ & $42.1(16.3)$ \\
\hline DBP mm Hg, mean (SD) & $83.8(11.2)$ & $92.0(11.4)$ & $93.2(12.0)$ & $86.7(8.5)$ \\
\hline SBP mm Hg, mean (SD) & $134.9(20.5)$ & $151.6(22.7)$ & $153.4(23.8)$ & $137.4(17.1)$ \\
\hline Men, $\%$ & 46.8 & 51.2 & 49.4 & 55.6 \\
\hline
\end{tabular}

LADA, latent autoimmune diabetes in adults; DBP, diastolic blood pressure; SBP, systolic blood pressure

From both of these investigations data are available for a cohort of 38,805 men and women, who were free of diabetes at baseline (i.e. at the time of the first health survey between 1984 and 1986) and for whom baseline information on smoking, age, BMI and leisure-time physical activity is available.

The regional Ethical Committee approved these studies and all participants gave informed consent.

Identification and classification of diabetes. During the followup investigation between 1995 and 1997, the questionnaire identified 1130 new cases of diabetes. These subjects were given an appointment to have their fasting blood glucose measured together with levels of C-peptide and anti-GAD. Altogether $858(76 \%)$ patients completed this investigation. Information on treatment and complications was also collected and the diagnosis of diabetes verified by fasting blood glucose levels $(\geq 6.1 \mathrm{mmol} / \mathrm{l})$ and/or the subjects' medical history and treatment.

Patients starting insulin treatment within 6 months of diagnosis were classified as having type 1 diabetes, if, in addition, they were anti-GAD positive as defined below or had fasting C-peptide levels lower than $150 \mathrm{pmol} / \mathrm{l}(n=18)$. Patients were classified as having LADA if they were anti-GAD positive and had not been treated with insulin within 12 months of diagnosis $(n=81)$. Type 2 diabetes was defined as anti-GAD negative and no insulin treatment within 1 year of diagnosis $(n=738)$. In addition we classified three cases as gestational diabetes, three cases as maturity-onset diabetes of the young (MODY) and 14 cases as unclassifiable. One case was classified as having normal glucose tolerance.

Biochemical analysis. Anti-GAD and fasting C-peptide were analysed at the Hormone Laboratory of Aker University Hospital, Oslo. Anti-GAD was analysed by an immunoprecipitation radioligand assay based on a previously validated method [4]. Translation-labelled H3-GAD was used as tracer. Free and antibody-bound GAD were separated by protein-A coupled to Sepharose. The level of GAD antibodies was expressed as an index value relative to a standard serum. From the start of the assay in 1995 an index greater than 0.08 was considered as positive. The assay was tested for proficiency in a current diabetes autoantibody standardisation programme. The analyses were blinded, with 100 samples from control subjects and 50 samples from type 1 diabetic subjects being analysed. At the cut-off level of more than 0.08 , sensitivity was 0.64 and specificity 1.00. Analysis of C-peptide was by RIA (Diagnostic System Laboratories, Webster, Tex., USA).

Statistical analysis. We calculated odds ratios together with 95\% CIs using multiple logistic regression analysis (Proc Logistic, SAS/STAT; SAS Institute, Cary, N.C., USA). Confounding was tested for by inclusion of age, BMI, physical ac- tivity and alcohol consumption in the regression model. The odds ratios are referred to as relative risks (RR), since the incidence of diabetes during the follow-up was low enough to allow such an approximation.

\section{Results}

At baseline, subjects who developed type 2 diabetes and LADA were older and more obese than subjects who did not develop diabetes or those who developed type 1 diabetes (Table 1). There were no differences between type 2 diabetes and LADA patients with regard to age, BMI, blood pressure or sex distribution (Table 1).

Heavy ( $\geq 20$ cigarettes/day) and prolonged $(\geq 13$ pack-years) smoking was associated with an increased risk of type 2 diabetes after adjustment for age, sex and BMI (Table 2). Conversely, compared to subjects who had never smoked, the risk of developing LADA or traditional type 1 diabetes was substantially lower in current smokers. Additional adjustment for physical activity, education and alcohol consumption did not change any of these findings. For example, the relative risk of type 2 diabetes in heavy smokers was 1.53 , 95\% CI: 1.14-2.05, while current smokers had relative risks of $0.17,95 \% \mathrm{CI}: 0.04-0.78$ for type 1 diabetes and of $0.24,95 \%$ CI: $0.09-0.63$ for LADA.

Parameters of smoking at baseline were not significantly $(p>0.05)$ associated with fasting blood glucose and C-peptide at follow-up in subjects with type 2 diabetes or LADA, nor were titres of anti-GAD in LADA (data not shown).

\section{Discussion}

This study demonstrates strikingly opposite associations between smoking and type 2 diabetes on the one hand and smoking and autoimmune diabetes (LADA and traditional type 1 diabetes) on the other.

Incident cases of diabetes were identified by questionnaire. Although this method has been shown to be valid for identifying known cases of diabetes [5], cases of undiagnosed diabetes will be missed. Also noteworthy is the fact that our analysis was restricted to 
Table 2. Relative risk of type 2 diabetes, LADA and type 1 diabetes associated with smoking

\begin{tabular}{|c|c|c|c|c|c|c|c|c|c|c|}
\hline & 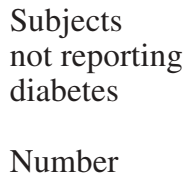 & \multicolumn{3}{|c|}{ Type 2 diabetes } & \multicolumn{3}{|l|}{ LADA } & \multicolumn{3}{|c|}{ Type 1 diabetes } \\
\hline \multicolumn{11}{|l|}{ Smoking } \\
\hline Current & 12,643 & 170 & 1.06 & $0.87-1.30$ & 6 & 0.25 & $0.11-0.60$ & 2 & 0.17 & $0.04-0.73$ \\
\hline \multicolumn{11}{|c|}{ Cigarettes per day (current smokers) } \\
\hline Never & 16,988 & 365 & 1.0 & & 50 & 1.0 & & 14 & 1.0 & \\
\hline$<20$ & 10,115 & 124 & 1.05 & $0.84-1.31$ & 4 & 0.21 & $0.08-0.60$ & 1 & 0.11 & $0.01-0.80$ \\
\hline$\geq 20$ & 1662 & 34 & 1.64 & $1.12-2.39$ & 1 & 0.28 & $0.04-2.05$ & 1 & 0.62 & $0.08-4.74$ \\
\hline$\geq 20$ & 2854 & 70 & 1.41 & $1.06-1.88$ & 4 & 0.49 & $0.17-1.41$ & 2 & 0.73 & $0.16-3.28$ \\
\hline \multicolumn{11}{|c|}{ Pack-years (former and current smokers) } \\
\hline Never & 16,988 & 365 & 1.0 & & 50 & 1.0 & & 14 & 1.0 & \\
\hline$<6$ & 6055 & 56 & 0.94 & $0.70-1.27$ & 7 & 0.80 & $0.35-1.83$ & 0 & & \\
\hline $6-12$ & 6075 & 81 & 1.05 & $0.81-1.36$ & 8 & 0.68 & $0.32-1.48$ & 3 & 0.52 & $0.15-1.82$ \\
\hline$\geq 13$ & 5733 & 164 & 1.38 & $1.12-1.69$ & 9 & 0.47 & $0.23-0.99$ & 1 & 0.21 & $0.03-1.65$ \\
\hline
\end{tabular}

Values are adjusted for age, sex and BMI. LADA, latent autoimmune diabetes in adults

diabetes diagnosed in adulthood, i.e. at 20 years of age or older (younger subjects were not included in the cohort). With regard, moreover, to the results for autoimmune diabetes, the greater number of LADA patients makes the findings stronger in this category than they would be in the category of traditional type 1 diabetes.

The question arises whether the associations found here between smoking and risk of diabetes could be secondary to other factors. Such a possibility is unlikely, since our results persisted after adjustment for age, BMI, physical activity, alcohol consumption and education. Still, we cannot completely rule out the possibility that residual confounding or lack of adjustment for factors such as diet or socio-economic situation could to some extent have contributed to our results.

Patients defined as having LADA are in most reports younger and less obese than those with type 2 diabetes (reviewed in [6]). We did not find such differences in the present study. The specificity of our anti-GAD assay seems to ensure that the patients classified as having LADA did indeed have autoimmune diabetes. Possible discrepancies between studies could be due to population differences, the presence or absence of other islet autoantibodies (not determined here) and anti-GAD epitope differences [6]. It should also be noted that we studied incident cases, whereas clinical characteristics of LADA have usually been reported from prevalence data.
An increased risk of type 2 diabetes in smokers has been attributed to increased insulin resistance [1], but an inhibitory effect of nicotine on insulin secretion has not been ruled out [7]. Additional associations between snuffing and type 2 diabetes [7] suggest that the diabetogenic effect of smoking in type 2 diabetes is linked to nicotine.

The association between smoking and autoimmune diabetes could be due to an inhibitory effect of nicotine on the immune system. Such an effect was recently demonstrated in the non-obese diabetic mouse, which is a model of autoimmune diabetes [8]. In humans, it has been shown that nicotinic receptors on leucocytes are increased in smokers [9], indicating that nicotine affects the immune system. The precise mechanisms behind the associations with smoking remain to be clarified.

What is the clinical significance of our findings? For many reasons they do not justify smoking to prevent autoimmune diabetes in adults. However, they constitute new knowledge that could be important for the prevention, by other means, of autoimmune-mediated destruction of beta cells.

Acknowledgements. The Nord-Trøndelag Health Study (The HUNT Study) is a collaboration between HUNT Research Centre, Faculty of Medicine, Norwegian University of Science and Technology (NTNU), Verdal, the Norwegian Institute of Public Health, and the Nord-Trøndelag County Council. GlaxoSmith Kline Norway and the Norwegian Diabetes Association also supported the diabetes part of the HUNT surveys. 


\section{References}

1. Eliasson B (2003) Cigarette smoking and diabetes. Prog Cardiovasc Dis 45:405-413

2. Midthjell K, Bjørndal A, Holmen J, Krüger $\varnothing$, Bjartveit K (1995) Prevalence of known and previously unknown diabetes mellitus and glucose intolerance in an adult Norwegian population: indications of an increasing diabetes prevalence; The Nord-Trøndelag Diabetes Study. Scand J Prim Health Care 13:229-235

3. Midthjell K, Krüger $\varnothing$, Holem J et al. (1999) Rapid changes in the prevalence of obesity and known diabetes in an adult Norwegian population. Diabetes Care 22:1813-1820

4. Petersen JS, Hejnaes KR, Moody A et al. (1994) Detection of GAD65 antibodies in diabetes and other autoimmune diseases using a simple radioligand assay. Diabetes 43: 459-467
5. Midthjell K, Holmen J, Bjørndal A, Lund-Larsen G (1992). Is questionnaire information valid in the study of a chronic disease such as diabetes? The Nord-Trøndelag diabetes study. J Epidemiol Community Health 46:537-542

6. Falorni A (2003) Immunologic and genetic aspects of latent autoimmune diabetes in the adult. Minerva Endocrinol 28:297-312

7. Persson P-G, Carlsson S, Efendic S, Östenson C-G, Grill V (2000) Cigarette smoking, oral moist snuff use and glucose intolerance. J Intern Med 248:103-110

8. Mabley JG, Pacher P, Southan GJ, Salzman AL, Szabo C (2002) Nicotine reduces the incidence of type 1 diabetes in mice. J Pharmacol Exp Ther 300:876-881

9. Benhammou K, Lee M, Strook M et al. (2000) ${ }^{3} \mathrm{H}$ nicotine binding in peripheral blood cells of smokers is correlated with the number of cigarettes smoked per day. Neuropharmacology 39:2818-2829 